\title{
The Effect of the Körner's Septum on Graft and Hearing Success after Type 1 Tympanoplasty
}

\author{
Mehmet Fatih Karakus, Suleyman Emre Karakurt, Kursat Murat Ozcan, Aykut Ikinciogullari, \\ Mustafa Colak and Haci Huseyin Dere \\ Department of Otorhinolaryngology, University of Health Sciences, Ankara Numune Training and Research Hospital, Ankara, Turkey
}

\begin{abstract}
Objective: To determine the effect of Körner's septum (KS) on graft success and hearing in patients who were treated with type 1 tympanoplasty.

Study Design: An observational study.

Place and Duration of Study: Department of Otorhinolaryngology, University of Health Sciences, Ankara Numune Training and Research Hospital, Turkey, from July 2013 to July 2018.

Methodology: Patients undergoing type 1 tympanoplasty were divided into 2 groups as patients with KS and without KS (WKS), according to the findings of high-resolution computed tomography of the temporal bone. Two different grafts (fascia and cartilage) in two groups (KS and WKS) were compared for graft success rate of different graft materials and pre- and postoperative hearing levels. The effect of the presence of KS on hearing was examined.

Results: The anatomical graft success rate of type 1 tympanoplasty was $75.9 \%$ in the KS group and $88.5 \%$ in the WKS group $(p=0.026)$. In the presence of KS, graft success rate decreased with the use of a fascia graft $(p=0.044)$. In the presence of KS, the use of cartilage graft did not affect functional hearing; whereas, poor functional outcomes were obtained in ears operated using a temporalis fascia graft in the KS group than in the WKS group $(p=0.003)$.

Conclusion: KS adversely affects graft success in type 1 tympanoplasty. Cartilage should be preferred as the graft material for patients with KS.
\end{abstract}

Key Words: Körner's septum, Tympanoplasty, Temporal fascia, Hearing, Cartilage.

How to cite this article: Karakus MF, Karakurt SE, Ozcan KM, Ikinciogullari A, Colak M, Dere HH. The effect of the Körner's septum on graft and hearing success after type 1 tympanoplasty. J Coll Physicians Surg Pak 2020; 30(2):154-157.

\section{INTRODUCTION}

During the pneumatisation of the temporal bone, the petrosquamous lamina normally disappears without any trace. However, Körner's septum (KS) occurs when a residue of this lamina remains as a developmental defect.1,2 KS is an osseous structure starting from the posterior portion of the mandibular fossa, running superior to the middle ear, vertically and inferiorly extending from the external side of the facial nerve canal, and ending at the mastoid apex. ${ }^{3} \mathrm{KS}$ is anatomically examined in three sections: Anterior temporomandibular, middle tympanic, and posterior mastoid sections. ${ }^{4}$ The posterior mastoid section of the $\mathrm{KS}$ is perpendicular to the part where the mastoid bone joins the temporosquamous section and can be examined in detail with high-resolution computed tomography (HRCT) of the temporal bone. ${ }^{5}$

The reduced surface area of the tympanic membrane during the co-occurrence of perforation has a negative

Correspondence to: Dr. Mehmet Fatih Karakus, Department of Otorhinolaryngology, University of Health Sciences, Ankara

Numune Training and Research Hospital, Ankara, Turkey.

E-mail:mfkarakus@yahoo.com

Received: November 05, 2019; Revised: December 21, 2019;

Accepted: January 24, 2020 effect on hearing due to the sound pressure passing into the middle ear. ${ }^{6}$ Type 1 tympanoplasty is a surgical procedure involving the closure of a tympanic membrane perforation using different materials. ${ }^{7}$ To the best of authors' knowledge, there are no studies that have investigated the effect of the presence of KS on graft success and hearing outcomes in type 1 tympanoplasty in the English literature.

The aim of this study was to compare anatomical and functional outcomes using two different graft materials between the groups of patients with and without KS who underwent type 1 tympanoplasty.

\section{METHODOLOGY}

This observational study was conducted at the Department of Otorhinolaryngology, University of Health Sciences, Ankara Numune Training and Research Hospital, Turkey from July 2013 to July 2018 . The study was approved by the local Ethics Committee and ethical principles of Helsinki Declaration conducted. Informed consent was obtained from all participants.

Two hundred and two cases who had central perforation of tympanic membrane and underwent type 1 tympanoplasty were included. Patients with choles-teatoma, 
adhesive otitis media, glomus tympanicum, ossicular chain disorders, and other accompanying ear/middle ear pathologies such as bilateral tympanic membrane perforation, marginal perforation, congenital anomalies detected on microscopy and HRCT of the temporal bone; those with inflamed/infected middle ear and mastoid mucosa; those operated for revision purposes; and those who underwent type 1 tympanoplasty with mastoidectomy or ossiculoplasty were excluded from the study.

Patients were divided into the following groups based on preoperative HRCT findings of the temporal bone: patients with KS (KS) and those without KS (WKS) (Figure 1). All operations were performed under general anesthesia using an operating microscope (MöllerWedel Optical; Hamburg, Germany) under the supervision of senior authors. The selection of graft material was made by the surgeon of the operation randomly. Regarding type 1 tympanoplasty, post-auricular approach used. A cartilage graft, prepared with an elevated unilateral perichondrium without thinning from the tragal cartilage, placed on the pericondrum on the inferior wall of the external auditory canal using an underlay technique. The prepared fascia graft was placed similarly. At the sixth postoperative month, anatomical success was evaluated with integrity based on otoscopic and microscopic findings. Pure-tone and speech audiometry measurements were performed before and at postoperative six months. Pure-tone averages (PTAs) were determined based on the threshold values at frequencies of 500,1000 , and $2000 \mathrm{kHz}$, and air-bone gap (ABG) PTA values were calculated. For audiometric tests, AC 40 clinical audiometer (AC 40; Interacuostic, Middelfart, Denmark) devices were used.

Shapiro-Wilk test was used to evaluate data distribution. Categorical variables are expressed as number (percentage); whereas, continuous variables are expressed as mean and standard deviation. Categorical variables were evaluated using Chi-square or Fisher's exact test. Differences between two independent groups were analysed using unpaired t-test or Mann-Whitney U-test. P-values $<0.05$ were considered significant.

\section{RESULTS}

Of the patients included in the study, age ranged $18-60$ years $(33.84 \pm 12.09), 51.9 \%$ of them were female. Patients were divided into two groups: KS (26.8\%) and WKS (73.2\%). Distribution of sex, age and graft materials were found to be similar in both groups.

The graft success rate was calculated to be $75.9 \%$ for the KS group and $88.5 \%$ for the WKS group. The presence of KS was found to decrease graft success rate $(p=0.026)$. When the ears treated using tragal cartilage; and temporalis fascia grafts were separately evaluated, graft success achieved in both groups were similar for tragal cartilage graft $(p=0.414)$. Graft success was achieved in the KS group treated with a temporalis fascia graft worse than the WKS group ( $p=0.044$; Table I).

In audiometric examination, the mean preoperative ABG PTA was found to be $28.2 \mathrm{~dB}$ for the KS group and $23.2 \mathrm{~dB}$ for the WKS group $(p=0.006)$. During the postoperative period, the mean $A B G$ difference (hearing gain) was found to be $14.0 \mathrm{~dB}$ in the KS group and 12.2 $\mathrm{dB}$ in the WKS group. The presence of KS had no postoperative effect on hearing gain $(p=0.194)$.



Figure 1: (A) Radiological apperance of Körner's septum (arrow) on axial HRCT images of right temporal bone.

(B) Radiological apperance of Körner's septum (arrow) on axial HRCT images of left temporal bone.

Table I: Graft success according to study groups.

\begin{tabular}{|c|c|c|c|c|c|c|}
\hline \multirow[t]{2}{*}{ Operated ear $(n=202)$} & \multicolumn{3}{|c|}{ With Korner's septum $(n=54)$} & \multicolumn{3}{|c|}{ Without Korner's septum $(n=148)$} \\
\hline & Fascia $(\mathrm{N}=26)$ & Cartilage $(\mathrm{N}=28)$ & $p$-value & Fascia $(\mathrm{N}=65)$ & Cartilage $(\mathrm{N}=83)$ & $\mathrm{p}$-value \\
\hline Graft success in groups, N (\%) & $16(61.5)$ & $25(89.3)$ & 0.017 & $53(81.5)$ & $78(94.0)$ & 0.019 \\
\hline Total graft success, N (\%) & $41(75.9)$ & & & $131(88.5)$ & & 0.026 \\
\hline Total cartilage grafts, N (\%) & - & $25(89.3)$ & - & & $78(94.0)$ & 0.414 \\
\hline Total fascia grafts, $\mathrm{N}(\%)$ & $16(61.5)$ & - & & $53(81.5)$ & - & 0.044 \\
\hline
\end{tabular}

Bold value signifies level was $0.05 . \quad N=$ Number of ears.

Table II: Functional audiologic findings (pure tone average, $\mathrm{dB}$ ).

\begin{tabular}{|c|c|c|c|c|c|c|c|}
\hline \multirow{2}{*}{$\begin{array}{l}\text { Postoperated } \\
\text { ABG }\end{array}$} & \multicolumn{3}{|c|}{ With Korner's septum $(n=54)$} & \multicolumn{3}{|c|}{ Without Korner's septum $(n=148)$} & \multirow[t]{2}{*}{$\mathrm{p}$-value } \\
\hline & Fascia $(\mathrm{N}=26)$ & Cartilage $(\mathrm{N}=28)$ & Total N (\%) & Fascia $(\mathrm{N}=65)$ & Cartilage $(\mathrm{N}=83)$ & Total N (\%) & \\
\hline \multirow[t]{3}{*}{$0-10 \mathrm{~dB}, \mathrm{~N}(\%)$} & $8(30.8)$ & $19(67.9)$ & $27(50.0)$ & $42(64.6)$ & $49(59.0)$ & $91(61.5)$ & 0.143 \\
\hline & - & $19(67.9)$ & - & & $49(59.0)$ & & 0.407 \\
\hline & $8(30.8)$ & - & & $42(64.6)$ & - & & 0.003 \\
\hline
\end{tabular}

Bold value signifies level was $0.05 ; \quad d B=$ Decibel; $\quad N=$ Number of ears; $\quad A B G=$ Air bone gap. 
When postoperative $A B G$ interval $0-10 \mathrm{~dB}$ was considered as the success criterion, successful hearing was similar in both groups $(p=0.143) .{ }^{8}$ The functional hearing outcomes of tragal cartilage was comparable in groups $(p=0.407)$. For temporalis fascia grafts, functional hearing success was better in the WKS group $(p=0.003$; Table II).

\section{DISCUSSION}

This is probably the first study in the English literature that investigated the relationship between the presence of $\mathrm{KS}$ and anatomical graft success to the best of authors' knowledge. It was seen that the presence of KS significantly reduced the success rate of temporalis fascia grafts, but not tragal cartilage grafts.

The incidence of KS has been reported to be as high as $30.4 \%$ in patients with adhesive otitis media, and $17.4 \%$ in patients with varying degrees of middle ear and mastoid air cell involvement. ${ }^{1}$ In a retrospective study of 356 HRCT examinations, this rate was found to be $31.0 \%$ in well-pneumatised mastoids and $25.9 \%$ in poorly pneumatised mastoids. ${ }^{3}$ In the present study, the incidence of $\mathrm{KS}$ was $26.8 \%$ in patients with intact ossicular chain, normal middle ear mucosa, and mastoid aeration without an evidence of retraction pockets or adhesive otitis media.

Relatively low success rates of temporalis fascia grafts, widely used in primary tympanoplasty for eustachian tube dysfunction, retraction pockets, adhesive otitis media, and wide perforations, have recently increased the popularity of cartilage grafts made of rigid materials more resistant to retraction, resorption, and infection.9,10 In a study comparing the anatomical success rate of temporal fascia and tragal cartilage graft materials used in type 1 tympanoplasty, the success rate was $80.6 \%$ in the temporal fascia group and $88.5 \%$ in the tragal cartilage group. 10 Khan et al. found that anatomical success rate at postoperative 2-year follow-up was $87.4 \%$ for the temporal fascia group and $98.2 \%$ for the tragal cartilage group. ${ }^{11}$ In a meta-analysis, the mean success rate of temporalis fascia tympanoplasty was $82 \%$ and that of different cartilage grafts was $92 \%$, indicating that cartilage graft success was significantly higher. ${ }^{7}$ In our study, the success rate with a tragal cartilage graft was higher in both groups.

In a study that investigated operation and patientinduced prognostic factors affecting surgical success and hearing outcomes, it was found that the success rates were significantly higher among non-smokers, in ears that remained dry for a long time, in healthy contralateral ears and when operation was performed by an experienced surgeon. ${ }^{12}$ Similarly, in another study that investigated the preoperative prognostic factors for the outcomes of type 1 tympanoplasty, it was reported that age $(<16$ years), presence of adenoid vegetation, and diseased contralateral ears had a prognostic role in graft success; that these factors decreased the success rate with a temporal fascia graft; and that there was no effect on cartilage graft success. ${ }^{13}$ In this study, the success rate of temporalis fascia graft was found to be low in the presence of KS. It is believed that this condition can be considered a prognostic factor for type 1 tympanoplasty.

In a meta-analysis by Jalali et al.7 there was no difference between the cartilage graft and temporal fascia graft groups when $A B G$ values below $10 \mathrm{~dB}$ were compared. When mean postoperative $A B G$ values were compared in a different meta-analysis, although there was no difference in terms of hearing gain, it was noted that hearing outcomes were better in the full thickness cartilage graft sub-group than in the temporal fascia group. 9

In studies on functional hearing outcomes, the incidence rate of cases with mean $A B G$ values of $10 \mathrm{~dB}$ and higher during the postoperative period was reported to be $57.7 \%$ by Patil et al., ${ }^{14} 69.0 \%$ by Guler et al, 15 and $76 \%$ by Dapholkar et al. ${ }^{16}$ for temporal fascia graft material. For tragal cartilage graft material, these values were $54.3 \%, 61.3 \%$ and $75 \%$, respectively. ${ }^{14-16}$ When hearing outcomes were evaluated using mean postoperative $A B G$ values of $10 \mathrm{~dB}$ and higher, it was found that this rate was $54.9 \%$ for the temporalis fascia group and $61.2 \%$ for the tragal cartilage group. The presence of KS had no significant effect on functional hearing.

It was found that the use of tragal cartilage as a graft material in the presence of KS did not result in a significant change in functional hearing outcomes; in the case when a temporalis fascia graft was used and when postoperative functional success was significantly lower. Similarly, it was observed that the anatomical success rate of temporalis fascia grafts was significantly lower in the same group of ears, and the results were consistent with each other.

\section{CONCLUSION}

Cartilage graft materials should be preferred in patients scheduled for type 1 tympanoplasty who are found to have KS on HRCT of the temporal bone. The presence of $\mathrm{KS}$ on $\mathrm{HRCT}$, a procedure that is now almost routinely used for preoperative imaging, plays a prognostic role in predicting graft success.

\section{ETHICAL APPROVAL:}

Ethics Committee approval was received for this study from the Ethics Committee of University of Health Sciences, Ankara Numune Training and Research Hospital (Number: E-18-2319, November 29, 2018).

\section{PATIENTS' CONSENT:}

Informed consents were obtained from all individual participants included in the study. 


\section{CONFLICT OF INTEREST:}

Authors declared no conflict of interest.

\section{AUTHORS' CONTRIBUTION:}

MFK: Contributed to the study design, revised it critically for important intellectual content, data acquisition and paper writing.

SEK: Contributed to the study design, data acquisition and data analysis.

KMO: Revised it critically for important intellectual content and data acquisition.

Al: Data acquisition and revised it critically for important intellectual content.

MC: Data acquisition and data analysis.

HHD: Data acquisition and revised it critically for important intellectual content.

\section{REFERENCES}

1. Göksu N, Kemaloglu YK, Köybasioglu A, lleri F, Ozbilen S, Akyildiz N. Clinical importance of the Korner's septum. Am J Otol 1997; 18:304-6.

2. Virapongse C, Sarwar M, Bhimani S, Sasaki C, Shapiro R. Computed tomography of temporal bone pneumatization: Petrosquamosal suture and septum. Am J Neuroradiol 1985; 6:561-8.

3. Toros SZ, Karaca CT, Habesoglu TE, Noseri H, Ertugay CK, Naiboglu $\mathrm{B}$, et al. Is there a relation between mastoid aeration and Körner's septum? Eur Arch Otorhinolaryngol 2010; 267:1523-6.

4. Virapongse C, Kirchner JC, Sasaki C, Shapiro M. Computed tomography of Körner's septum and pertosquamosal suture. Arch Otolaryngol Head Neck Surg 1986; 112:81-7.

5. Ozer E, Bayazit YA, Kara C, Mumbuç S, Kanlikama M, Gümüsburun E. Körner's septum (petrosquamosal lamina) and chronic ear disease. Surg Radiol Anat 2004; 26:118-21.

6. lacovou E, Vlastarakos PV, Panagiotakopoulou A, Chrysostomou M, Kandiloros D, Adamopoulos G, et al. Effect of type I tympano- plasty on the resonant frequency of the middle ear: Comparison between chondrotympanoplasty and temporalis fascia grafting. J Otolaryngol Head Neck Surg 2012; 41:14-9.

7. Jalali MM, Motasaddi M, Kouhi A, Dabiri S, Soleimani R. Comparison of cartilage with temporalis fascia tympanoplasty: A meta-analysis of comparative studies. Laryngoscope 2017; 127:2139-48.

8. Monsell EM. New and revised reporting guidelines from the committee on hearing and equilibrium. American academy of otolaryngology - head and neck surgery foundation, inc. Otolaryngol Head Neck Surg 1995; 113:176-8.

9. Yang $\mathrm{T}$, Wu X, Peng X, Zhang $\mathrm{Y}$, Xie S, Sun H. Comparison of cartilage graft and fascia in type 1 tympanoplasty: Systematic review and meta-analysis. Acta Otolaryngol 2016; 136:1085-90.

10. Sözen E, Orhan Uçal Y, Tansuker HD, Uslu Corkun B, Yasemin Korkut A, Dadas B. Is the tragal cartilage necessary for type 1 tympanoplasties? J Craniofac Surg 2012; 23:e280-3.

11. Khan MM, Parab SR. Comparative study of sliced tragal cartilage and temporalis fascia in type I tympanoplasty. $J$ Laryngol Otol 2015; 129:16-22.

12. Onal K, Uguz MZ, Kazikdas KC, Gursoy ST, Gokce H. A multivariate analysis of otological, surgical and patient-related factors in determining success in myringoplasty. Clin Otolaryngol 2005; 30:115-20.

13. Salviz M, Bayram O, Bayram AA, Balikci HH, Chatzi T, Paltura C, et al. Prognostic factors in type I tympanoplasty. Auris Nasus Larynx 2015; 42:20-3.

14. Patil T, Baisakhiya N, Deshmukh PT. Evaluation of different graft material in type 1 tympanoplasty. Indian J Otology 2014; 20:106-14.

15. Guler I, Baklaci D, Kuzucu I, Kum RO, Ozcan M. Comparison of temporalis fascia and tragal cartilage grafts in type 1 tympanoplasty in elderly patients. Auris Nasus Larynx 2019; 46:319-23.

16. Dabholkar JP, Vora K, Sikdar A. Comparative study of underlay tympanoplasty with temporalis fascia and tragal perichondrium. Indian J Otolaryngol Head Neck Surg 2007; 59:116-9. 